\title{
An Underwater Acoustic Telemetry Modem for Eco-Sensing
}

\author{
Ronald A. Iltis, Hua Lee, Ryan Kastner, \\ Daniel Doonan, Tricia Fu, Rachael Moore and Maurice Chin \\ Department of Electrical and Computer Engineering \\ University of California, \\ Santa Barbara, CA 93106-9560 \\ Tel: (805) 893-4166, E-mail: \{iltis,kastner,lee\}@ece.ucsb.edu
}

\begin{abstract}
An underwater acoustic telemetry modem is presented for ecological research (Eco-sensing) applications. The modem is intended for the physical layer (PHY) in an Ad hoc network of "AquaNodes". Each AquaNode will incorporate an acoustic modem, router and host processor with standardized interfaces to oceanographic sensors. The transmitted waveforms use a composite Walsh/m-sequence format in which each bit in an 8-bit long Walsh function is spread by a 7-chip m-sequence. The resulting waveform has a $5 \mathrm{kHz}$ bandwidth for robustness to multipath and achieves a $\mathbf{1 3 3}$ bps data rate. An 11 msec. time-guard band for channel clearing eliminates the need for equalization. The receiver employs a Generalized Multiple Hypothesis Test with a Matching Pursuits (GMHT-MP) inner loop for symbol-by-symbol channel estimation. Symbol-error rate (SER) results demonstrate that the GMHT-MP is superior to the noncoherent RAKE receiver at usable SERs $\left(<10^{-2}\right)$ and can accommodate up to 10 msec. multipath spreads with Doppler spreads on the order of $1 \mathrm{~Hz}$.
\end{abstract}

\section{INTRODUCTION}

Underwater Eco-Sensing deals with telemetry of marine environmental data (conductivity, temperature, depth, currents). There is a great need for undersea wireless networks for Eco-Sensing allowing remote monitoring and adaptive sampling without requiring physical retrieval of instruments. However, underwater wireless networks developed so far for telemetry [1][2] rely on expensive, high power acoustic modems typically based on M-ary FSK [3][4]. The fundamental obstacle to robust UWA communications is multipath which introduces severe intersymbol interference (ISI). MFSK modems use narrowband tones with duration much greater than the multipath spread, thus eliminating ISI. Alternatively, equalizers [5] and direct-sequence spreadspectrum [6][7][8] modulation have been employed to reduce the effects of ISI and frequency-selective multipath.

An alternative underwater acoustic (UWA) modem using direct-sequence signaling is presented here based on composite Walsh and m-sequence waveforms. The advantage of such Walsh/m-sequence signaling is that the waveform is instantaneously wideband, in contrast to M-FSK, thus providing robustness to frequency-selective multipath. The application considered is underwater telemetry in a short-range $(<500 \mathrm{~m})$ shallow water channel, where multipath spreads can easily extend to 10 msec. [7][9]. The target bit rate is $133 \mathrm{bps}$, which is adequate for conductivity/temperature/depth (CTD) measurements in Eco-Sensing applications. The UWA modem will be incorporated in "AquaNodes" to form an Ad hoc UWA network. Each AquaNode will contain a host processor, router, and standard sensor interfaces for deployment in a wide range of Long Term Ecological Research (LTER) sites including coral reefs, lakes and coastal areas.

The notation and parameters employed are as follows. $\mathbf{x}$ is a column vector, $\mathbf{M}$ is a matrix, $(\mathbf{x})_{i}$ is the $i$-th element of a vector, and $(\mathbf{M})_{i, j}$ is the $i, j$-th matrix element. Where necessary, Matlab notation is employed with $\mathbf{M}(:(i), i(:))$ denoting the $i$-th column (row) of a matrix for example. The system parameters are

Chip duration: $T_{c}=.2 \mathrm{msec}$.

Sampling interval: $T_{s}=T_{c} / 2=.1 \mathrm{msec}$. m-sequence length: $L_{p n}=7$ chips.

Walsh sequence length: $N_{w}=8$ binary symbols Symbol duration: $T_{\text {sym }}=L_{p n} N_{w} T_{c}=11.2 \mathrm{msec}$. Samples/symbol: $N_{s}=T_{\text {sym }} / T_{s}=112$.

Time guard interval: $T_{g}=T_{\text {sym }}$ sec. per symbol. Bit rate: $R_{b}=(3$ bits $/$ symbol $) /\left(T_{\text {sym }}+T_{g}\right)=133$ bps.

Binary Walsh sequences: $\mathbf{w}_{\mathrm{m}} \in\{ \pm 1\}^{N_{W}}$ for $\mathrm{m}=$ 
$1, \ldots, N_{W}$.

m-sequence: $\mathbf{c} \in\{ \pm 1\}^{L_{p n}}$.

Transmitted Walsh/m-sequence: $\mathbf{d}_{\mathrm{m}}=\mathbf{w}_{\mathrm{m}} \otimes \mathbf{c} \in$ $\{ \pm 1\}^{N_{W} L_{p n}}$, where $\otimes$ is the Kronecker product.

Complex multipath gains: $\alpha_{1}(n), \ldots, \alpha_{N_{\alpha}}(n) \in \mathbb{C}$.

Path delays: $\tau_{1}(n)<\tau_{2}(n) \ldots<\tau_{N_{\alpha}}(n) \in$ $\left[0, T_{\text {sym }}\right]$.

The Walsh/m-sequence transmitted waveform is shown in Fig. 1. The Walsh sequences are generated via the recursion of Hadamard matrices $\mathbf{H}^{N_{W}}=\mathbf{H}^{N_{W} / 2} \otimes$ $\mathbf{H}^{2}$ where $\mathbf{H}_{2}=[11 ; 1-1]$ (Matlab notation) and $\mathbf{H}^{1}=1$. Hence the $\mathrm{m}$-th sequence is the $\mathrm{m}$-th row of the Walsh matrix $\mathbf{w}_{\mathrm{m}}=\mathbf{H}^{N_{W}}(\mathrm{~m},:)$. The specific $\mathrm{m}$-sequence employed is $\mathbf{c}=[1,1,-1,1,-1,-1,-1]^{T}$. The transmitted waveform is then

$$
s_{T}(t)=\sum_{n=0}^{\infty}\left(\mathbf{d}_{\mathrm{m}(n)}\right){ }_{i} g\left(t-i T_{c}-2 n T_{s y m}\right),
$$

where $g(t)$ is a raised-cosine pulse with bandwidth $\beta / T_{c}$, $.5<\beta \leq 1$ and $\mathrm{m}(n)$ is the symbol transmitted in epoch $\left[2 n T_{\text {sym }},(2 n+1) T_{\text {sym }}\right)$. Note that the sampling interval $T_{s}=T_{c} / 2$ corresponds to an oversampling rate of two for zero percent excess bandwidth.

\section{Channel Model And Generalized Multiple HYPOTHESIS TEST}

The received waveform in $t \in\left[2 n T_{\text {sym }}, 2(n+1) T_{\text {sym }}\right)$ is given by

$$
r(t)=\sum_{p=1}^{N_{\alpha}} \alpha_{p}(n) s_{\mathrm{m}(n)}\left(t-\tau_{p}(n)\right)+n(t),
$$

where

$$
s_{\mathrm{m}(n)}(t)=\sum_{i=0}^{N_{w} L_{p n}-1}\left(\mathbf{d}_{\mathrm{m}(n)}\right)_{i} g\left(t-i T_{c}\right),
$$

and $n(t) \in \mathcal{C}$ is additive wideband receiver/ambient noise. The duration of $s_{\mathrm{m}}(t)$ is $T_{\text {sym }}=2 N_{W} L_{p n} T_{s}$ sec., hence $r(t)$ in (2) includes a $T_{g}=T_{\text {sym }}$ sec. time-guard interval. Under the assumptions that $0 \leq \tau_{p}(n) \leq T_{\text {sym }}$, and coarse symbol acquisition has been achieved, intersymbol interference is thus eliminated in (2). (Symbol sync is discussed in Section IV.) The noise $n(t)$ is approximated as circular white Gaussian with spectral density $N_{0}$. Thus, assuming bandlimiting to $1 / T_{c}=$ $2 / T_{s} \mathrm{~Hz}$., the variance of $n(t)$ is $E\left\{|n(t)|^{2}\right\}=2 N_{0} / T_{s}$. The channel parameters are assumed constant in the interval $2 T_{\text {sym }}$ seconds, but independent from symbolto-symbol hence the $\alpha_{p}(n), \tau_{p}(n)$ vary with epoch $n$.
In order to simplify the receiver and subsequent presentation, an approximate tapped-delay line model for the multipath is assumed [10]. Thus

$$
r(t) \approx \sum_{l=0}^{N_{s}-1} f_{l} s_{\mathrm{m}(n)}\left(t-l T_{s}\right)+n(t) .
$$

where $f_{l} \in \mathbb{C}$ are the coefficients from an interpolation formula approximation to the true channel. Since the channel is typically sparse, the $f_{l}$ will be estimated under the constraint that only $N_{f}<<N_{s}$ coefficients are nonzero. The received signal is sampled at rate $1 / T_{s}$ over an interval $2 T_{\text {sym }}$ to include the time-guard interval. The resulting received vector model is then

$$
\mathbf{r}(n) \approx \sum_{l=0}^{N_{s}-1} f_{l} \mathbf{s}_{\mathrm{m}(n)}(l)+\mathbf{n}(n),
$$

where $\mathbf{r}(n), \mathbf{s}_{\mathrm{m}(n)}(l) \in \mathbb{C}^{2 N_{s}}$ are

$\mathbf{r}(n)=$

$$
\begin{aligned}
& {\left[r\left(\left(2(n+1) N_{s}-1\right) T_{s}\right) r\left(\left(2(n+1) N_{s}-2\right) T_{s}\right) \ldots\right.} \\
& \left.r\left(2 n N_{s} T_{s}\right)\right]^{T} \\
& \mathbf{s}_{\mathrm{m}(n)}(l)= \\
& {\left[s_{\mathrm{m}(n)}\left(\left(2 N_{s}-1-l\right) T_{s}\right) s_{\mathrm{m}}\left(\left(2 N_{s}-2-l\right) T_{s}\right)\right.} \\
& \left.\ldots, s_{\mathrm{m}(n)}\left(-l T_{s}\right)\right]^{T} .
\end{aligned}
$$

The channel coefficients $f_{l}(n)$ are unknown and may be rapidly varying (e.g. Doppler spreads of $.5 \mathrm{~Hz}$ are reported in [11].) Channel estimation techniques such as Kalman filtering are too complex for implementation in the envisioned low-cost telemetry modem. Here, a Generalized Multiple Hypothesis Test receiver is proposed, using the Matching Pursuits algorithm [12][13] for instantaneous channel estimation. The GMHT is compactly expressed as the decision rule (dropping the time index)

$$
\hat{\mathbf{m}}=\arg \min _{\mathbf{m}}\left\{\min _{\mathbf{f}:|\mathbf{f}|=N_{f}}\left\|\mathbf{r}-\mathbf{S}_{\mathbf{m}} \mathbf{f}\right\|^{2}\right\},
$$

where $\mathbf{f}=\left[f_{0} f_{1} \ldots f_{N_{s}-1}\right]^{T}$. The constraint $|\mathbf{f}|=N_{f}$ indicates that at most $N_{f}<<N_{s}$ elements of $\mathbf{f}$ are nonzero. The signal matrix $\mathbf{S}_{n} \in \mathbb{C}^{2 N_{s} \times N_{s}}$ is

$$
\mathbf{S}_{\mathrm{m}}=\left[\mathbf{s}_{\mathrm{m}}(0) \mathbf{s}_{\mathrm{m}}(1) \ldots \mathbf{s}_{\mathrm{m}}\left(N_{s}-1\right)\right] .
$$

Unfortunately, the exact minimization over $\mathbf{f}$ in the GMHT (7) has complexity growing exponentially in $N_{s}$. Hence, the GMHT is replaced by

$$
\hat{\mathbf{m}}=\arg \min _{\mathrm{m}}\left\{\left\|\mathbf{r}-\mathbf{S}_{\mathrm{m}} \hat{\mathbf{f}}_{\mathrm{m}}^{M P}\right\|^{2}\right\},
$$


where $\hat{\mathbf{f}}_{\mathrm{m}}^{M P}$ is the Matching Pursuits estimate of the channel conditioned on the $\mathrm{m}$-th Walsh sequence being transmitted. In the sequel, $\hat{\mathbf{f}}_{\mathrm{m}}$ will always refer to the sequence conditional MP estimate.

\section{GMHT-MP RECEIVER}

The Matching Pursuits algorithm is described next. It is computationally efficient to employ a sufficient statistics representation of the cost function in (9) as follows:

$$
-\left\|\mathbf{r}-\mathbf{S}_{\mathbf{m}} \hat{\mathbf{f}}\right\|^{2} \propto 2 R e\left\{\mathbf{v}_{\mathrm{m}}^{H} \hat{\mathbf{f}}\right\}-\hat{\mathbf{f}}^{H} \mathbf{A}_{\mathrm{m}} \hat{\mathbf{f}},
$$

where $\mathbf{v}_{\mathrm{m}}=\mathbf{S}_{\mathrm{m}}^{H} \mathbf{r}$ and $\mathbf{A}_{\mathrm{m}}=\mathbf{S}_{\mathrm{m}}^{H} \mathbf{S}_{\mathrm{m}}$.

Recall that the problem is to estimate $\mathbf{f} \in \mathbb{C}^{N_{s}}$ under the constraint that only $|\mathbf{f}|=N_{f}<<N_{s}$ coefficients are nonzero. In order to obtain such a sparse solution, MP iteratively updates the assumed channel order and estimates the next coefficient using a canceled signal. Specifically, at iteration $k=1$ of MP, it is assumed that $N_{f}=1$ and the index of the detected path is [12]

$$
q_{1}=\arg \max _{i}\left(2 \operatorname{Re}\left\{\left(\mathbf{v}_{\mathrm{m}}\right)_{i}^{*} \hat{\mathbf{f}}_{i}\right\}-\left(\mathbf{A}_{\mathrm{m}}\right)_{i, i}\left|\hat{f}_{i}\right|^{2}\right) .
$$

The estimate $\hat{\mathbf{f}}_{i}$ is

$$
\hat{\mathbf{f}}_{i}=\arg \max _{f} 2 \operatorname{Re}\left\{\left(\mathbf{v}_{\mathrm{m}}\right)_{i}^{*} f\right\}-\left(\mathbf{A}_{\mathrm{m}}\right)_{i, i} f=\frac{\left(\mathbf{v}_{\mathrm{m}}\right)_{i}}{\left(\mathbf{A}_{\mathrm{m}}\right)_{i, i}} .
$$

Thus, the detected index on MP iteration $k=1$ reduces to $q_{1}=\arg \max _{i}\left|\left(\mathbf{v}_{\mathrm{m}}\right)_{i}\right|^{2} /\left(\mathbf{A}_{\mathrm{m}}\right)_{i, i}$.

At stage $k$ of MP, a canceled signal is formed as

$$
\mathbf{r}^{k}=\mathbf{r}-\sum_{i=1}^{k-1} \mathbf{S}_{\mathrm{m}}\left(:, q_{i}\right) \hat{f}_{q_{i}} .
$$

The signal $\mathbf{r}^{k}$ is then used to detect the next path index $q_{k}$ [12][13]. The sufficient statistic becomes

$$
\mathbf{v}_{\mathrm{m}}^{k}=\mathbf{S}_{\mathrm{m}}^{H} \mathbf{r}^{k}=\mathbf{v}_{\mathrm{m}}-\sum_{i=1}^{k-1} \mathbf{A}_{\mathrm{m}}\left(:, q_{i}\right) \hat{f}_{q_{i}} .
$$

Using (14), the $k$-th detected coefficient index is

$$
q_{k}=\arg \max _{i \neq q_{1}, \ldots, q_{k-1}} \frac{\left|\left(\mathbf{v}_{\mathrm{m}}^{k}\right)_{i}\right|^{2}}{\left(\mathbf{A}_{\mathrm{m}}\right)_{i, i}} .
$$

Note that $\left(\mathbf{A}_{\mathrm{m}}\right)_{i, i}$ is not required in eq. (15) for the equalenergy signal set employed here. Table I summarizes the combination of Matching Pursuits with the GMHT.

$$
\begin{aligned}
& \text { For } \mathrm{m}=1,2, \ldots N_{W} \\
& \text { Get current received vector } \mathbf{r} \\
& \mathbf{v}_{\mathrm{m}}^{1}=\mathbf{S}_{\mathrm{m}}^{H} \mathbf{r} \\
& \text { Initialize channel estimate } \hat{\mathbf{f}}_{\mathrm{m}}=\mathbf{0} \\
& \text { For } k=1,2, \ldots, N_{f} \\
& \quad q_{k}=\arg \max _{i \neq q_{1}, \ldots, q_{k-1}}\left|\left(\mathbf{v}_{\mathrm{m}}^{k}\right)_{i}\right|^{2} /\left(\mathbf{A}_{\mathrm{m}}\right)_{i, i} \\
& \quad\left(\hat{\mathbf{f}}_{\mathrm{m}}\right)_{q_{k}}=\left(\mathbf{v}_{\mathrm{m}}^{k}\right)_{q_{k}} /\left(\mathbf{A}_{\mathrm{m}}\right)_{q_{k}, q_{k}} \\
& \quad \mathbf{v}_{\mathrm{m}}^{k+1}=\mathbf{v}_{\mathrm{m}}^{k}-\mathbf{A}_{\mathrm{m}}\left(:, q_{k}\right)\left(\hat{\mathbf{f}}_{\mathrm{m}}\right)_{q_{k}} \\
& \text { Next } k
\end{aligned}
$$

Next $m$

Make transmitted symbol decision using GMHT

$\hat{\mathbf{m}}=\arg \max _{\mathrm{m}} 2 \operatorname{Re}\left\{\mathbf{v}_{\mathrm{m}}^{H} \hat{\mathbf{f}}_{\mathrm{m}}\right\}-\hat{\mathbf{f}}_{\mathrm{m}}^{H} \mathbf{A}_{\mathrm{m}} \hat{\mathbf{f}}_{\mathrm{m}}$

\section{TABLE I}

GMHT-MP ALGORITHM FOR THE UWA MODEM.

\section{Symbol Synchronization Algorithm}

The GMHT-MP algorithm in Table I assumes coarse symbol synchronization, such that the beginning of the vector $\mathbf{r}(n)$ for symbol $n$ is aligned with the direct path. In order to obtain symbol sync, a training sequence comprising $2 M N_{s}$ samples is first transmitted using $M$ known Walsh symbols with $\mathrm{m}(n)=1$ for $n=1, \ldots, M$. Recall that $2 N_{s}=2 T / T_{s}$ is the length in samples of the symbol plus time-guard interval. The synchronization algorithm first estimates the time-of-arrival of the direct path, and then advances the symbol clock appropriately.

Under the above assumptions, the received signal during the training period is approximated via a tappeddelay line multipath channel as

$$
r(t) \approx \sum_{l=0}^{2 N_{s}-1} f_{l} s_{1}\left(t-l T_{s}\right)+n(t) .
$$

The sparse channel with coefficients $f_{l}$ corresponds to unambiguous delays ranging from 0 to $\left(2 N_{s}-1\right) T_{s}$ s. The acquisition problem is then

1) Estimate the $\left\{f_{l}\right\}$ under a numerosity constraint $|\mathbf{f}|=N_{f}<<2 N_{s}$. Here, the channel vector is twice the length as in GMHT-MP, specifically $\mathbf{f}=$ $\left[f_{0} f_{2} \ldots f_{2 N_{s}-1}\right]^{T}$ and $|\mathbf{f}|$ is the number of nonzero elements in $\mathbf{f}$.

2) Find the index of the direct path $l_{d}$ in $\mathbf{f}$ such that the multipath channel is defined by $\left[f_{l_{d}}, f_{l_{d}+1 \bmod 2 N_{s}} \ldots f_{l_{d}+N_{s}-1 \bmod 2 N_{s}}\right]$. That is, if the direct-path delay is $2 N_{s}-1$ for example, and the next multipath coefficient is nonzero, the next coefficient will have associated delay 0 , due to the $2 N_{s}$ delay ambiguity. 
3) Collect vectors $\mathbf{r}(n)$ for subsequent demodulation with a timing advance of $l_{d}$ samples. This aligns the onset of each direct-path waveform $s_{1}(t)$ with the beginning of the $2 N_{s}$ sample window defined by $\mathbf{r}(n)$.

\section{Packet-Based Matching Pursuits}

The packet-based MP algorithm is defined somewhat differently from the GMHT-MP algorithm. First, since a training sequence is transmitted only one MP algorithm for $\mathrm{m}=1$ needs to be implemented. Second, a vector of length $2 M N_{s}$, rather than $N_{s}$ samples with $M>1$ is collected. The packet length $M$ is selected to achieve accurate initial channel estimates, but short enough so that the Doppler variation is negligible in $M 2 T_{\text {sym }}$ sec. Define the $n$-th received sample vector $\mathbf{r}(n)$ as

$$
\mathbf{r}(n)=\sum_{l=0}^{2 N_{s}-1} f_{l} \mathbf{s}_{1}(l)+\mathbf{n}(n),
$$

where with $r(n)=r_{c}\left(n T_{s}\right)$ and $r_{c}(t)$ the continuoustime received waveform, we have

$\mathbf{r}(n)=\left[r\left(2(n+1) N_{s}-1\right) r\left(2(n+1) N_{s}-2\right) \ldots r\left(2 n N_{s}\right)\right]^{T}$.

For training sequence transmission, the signal vector corresponds to

$$
\mathbf{s}_{1}(l)=\left[s_{1}\left(2 N_{s}-1-l\right) s_{1}\left(2 N_{s}-2-l\right) \ldots s_{1}(-l)\right]^{T} .
$$

However, unlike the GMHT-MP receiver, continual training sequence transmission results in a circular form for the vector $\mathbf{s}_{1}(l)$. Specifically, the $k$-th component $s_{1}(k)$ is given by

$$
s_{1}(k)=\sum_{q=0}^{1} s_{1}^{c}\left(k T_{s}+q 2 N_{s} T_{s}\right),
$$

where

$$
s_{1}^{c}(t)=\sum_{i=0}^{N_{w} L_{p n}-1}\left(\mathbf{d}_{1}\right)_{i} g\left(t-i T_{c}\right),
$$

where $\mathbf{d}_{1}=\mathbf{b}_{1} \otimes \mathbf{c}$ is the $N_{w}=8, L_{p n}=7$ or 56 chip long Walsh/m-sequence corresponding to training symbol $\mathrm{m}=1$. Thus, the sequence $s_{1}(k)$ is a periodic version of the $2 T_{\text {sym }}$ long symbol plus guard interval with period $2 N_{s}$. Hence $\mathbf{s}_{1}(l)$ in (19) is the $l$-th circular shift of the $2 N_{s}$ sample long Walsh/m-sequence waveform.

Next define a packet-length vector $\mathbf{r} \in \mathbb{C}^{2 M N_{s}}$ as

$$
\mathbf{r}=\left[\mathbf{r}(M-1)^{T} \mathbf{r}(M-2)^{T} \ldots \mathbf{r}(0)\right]^{T},
$$

Precompute $\mathbf{A}=\mathbf{S}^{H} \mathbf{S} \in \mathbb{C}^{2 N_{s} \times 2 N_{s}}$

Collect received packet $\mathbf{r} \in \mathbb{C}^{2 M N_{s}}$ over $2 M N_{s}$ samples $\mathbf{r}=\left[\mathbf{r}(M-1)^{T} \mathbf{r}(M-2) \ldots \mathbf{r}(0)\right]^{T}$

Initialize sufficient statistic vector $\mathbf{v}^{1}=\mathbf{S}^{H} \mathbf{r} \in \mathbb{C}^{2 N_{s}}$

For $k=1,2, \ldots, N_{f}$ $q_{k}=\arg \max _{i \neq q_{1}, \ldots, q_{k-1}}\left|\left(\mathbf{v}^{k}\right)_{i}\right|^{2} /(\mathbf{A})_{i, i}$ $\hat{f}_{q_{k}}=(\mathbf{v})_{q_{k}} /(\mathbf{A})_{q_{k}, q_{k}}$

Next $k$ $\mathbf{v}^{k+1}=\mathbf{v}^{k}-\mathbf{A}\left(:, q_{k}\right) \hat{f}_{q_{k}}$

Find direct-path index

$$
l_{d}=\arg \max _{l} \frac{\sum_{i=l}^{N_{s}+l-1}\left|\hat{f}_{i} \bmod 2 N_{s}\right|^{2}}{\sum_{i=N_{s}+l}^{2 N_{s}+l-1}\left|\hat{f}_{i} \bmod 2 N_{s}\right|^{2}}
$$

Advance subsequent timing for demodulation by $l_{d}$ samples

TABLE II

MP ALGORITHM FOR INITIAL TIMING/CHANNEL ESTIMATION

where $\mathbf{r}(n) \in \mathbb{C}^{2 N_{s}}$ is given by (18). Also define $\mathbf{S} \in$ $\mathbb{C}^{2 M N_{s} \times 2 N_{s}}$ with $i$-th column $\mathbf{S}_{i}=\mathbf{1}_{M} \otimes \mathbf{s}_{1}(i-1)$, where $\mathbf{1}_{M}$ is the length $M$ all-ones vector and $\otimes$ is the Kronecker product. Then the $i$-th column of $\mathbf{S}$ is the $i$ th circular shift of the transmitted waveform over $2 M N_{s}$ samples. The received packet is then

$$
\mathbf{r}=\mathbf{S f}+\mathbf{n}
$$

Using $\mathbf{r}$, the Matching Pursuits algorithm yields an estimate $\hat{\mathbf{f}}$ of the channel coefficients. Given $\hat{\mathbf{f}}$, we have to find the best-fit multipath channel such that the $N_{f}$ nonzero coefficients occupy at most $N_{s}$ contiguous samples, modulo $2 N_{s}$ starting at delay $l_{d}$. The following test for $l_{d}$ is proposed, under the assumption that the maximum multipath spread is $N_{s}$ samples, and hence at least $N_{s}$ contiguous coefficients (modulo $N_{s}$ ) $\hat{f}_{l}$ must be zero.

$$
l_{d}=\arg \max _{l} \frac{\sum_{i=l}^{N_{s}+l-1}\left|\hat{f}_{i \bmod 2 N_{s}}\right|^{2}}{\sum_{i=N_{s}+l}^{2 N_{s}+l-1}\left|\hat{f}_{i \bmod 2 N_{s}}\right|^{2}} .
$$

That is, we try to find the direct path delay which corresponds to the maximum of the ratio of the energy in the hypothesized multipath profile to the energy in the hypothesized set of $N_{s}$ zero coefficients $\hat{f}_{l}$.

The overall synchronization/channel estimation algorithm is summarized in Table II.

\section{RESUlTS}

The GMHT-MP algorithm was simulated using independent Rayleigh fading channels $\alpha_{p}(n)$ from symbol- 
to-symbol. The delays $\tau_{p}(n)$ were generated as independent random variables uniform on $\left[0, T_{\text {sym }}\right]$ on each epoch $n$.

An example of instantaneous channel estimation using one symbol epoch of data, $\mathbf{r}(n)$ is shown in Fig. 2. The true channel multipath intensity profile (MIP) is plotted along with the estimated channel MIP using $\hat{\mathbf{f}}$. In this case, there are $N_{\alpha}=6$ actual paths, and Matching Pursuits assumes $N_{f}=8$ paths are present. The Matching Pursuits channel estimator clearly identifies the major paths using just one symbol epoch of data.

The SERs for the GMHT-MP and RAKE receivers are plotted in Fig. 3 for randomly generated channels on each symbol. Note that the RAKE detector corresponds to GMHT-MP in Table I with the cancellation step removed, i.e. with $\mathbf{v}_{\mathrm{m}}^{k}=\mathbf{S}_{\mathrm{m}}^{H} \mathbf{r}(n)$ at each iteration. Again, the $\alpha_{p}$ were chosen as i.i.d. zero-mean circular Gaussian r.v.s (Rayleigh fading), such that the channel energy gain was normalized to unity. The noncoherent combining loss and fading impacts the SER so that an $E_{b} / N_{0}$ of $20 \mathrm{~dB}$ is required to obtain a $10^{-3}$ SER. It is seen that the GMHT-MP receiver significantly outperforms the RAKE at usable SERs below $10^{-2}$. This result is not surprising, since as SNR increases, the GMHT-MP channel estimates become increasingly accurate, and the receiver approaches the coherent ML detector. In contrast, the RAKE performance is limited to that of a noncoherent detector. Finally, observe that the GMHT-MP performance is not adversely affected by channel overparameterization (e.g. $N_{\alpha}=32$ with $N_{f}=48$ assumed.

An example run from the synchronization algorithm in Table II is shown in Fig. 5. The estimated MIP closely follows the true MIP, and the algorithm correctly detects the time-of-arrival of the direct path with delay $l_{d}$ samples.

\section{HARDWARE IMPLEMENTATION}

The GMHT-MP modem is currently being implemented at UCSB using the TI F2812 fixed-point DSP device with custom amplifiers, matching networks and transducers. The GMHT-MP real-time software has been successfully tested in the lab and verified using Code Composer. The F2812 board with amplifier/matching module is shown in Fig. 4.

In the receive mode, the signal from the $25 \mathrm{kHz}$ center frequency transducer is amplified and filtered, with a large gain-adjust range in the amplifiers $(84 \mathrm{~dB}$ ) allowing adaptation to a wide range of conditions. The filtered signal is applied to the 12-bit ADC which is a module

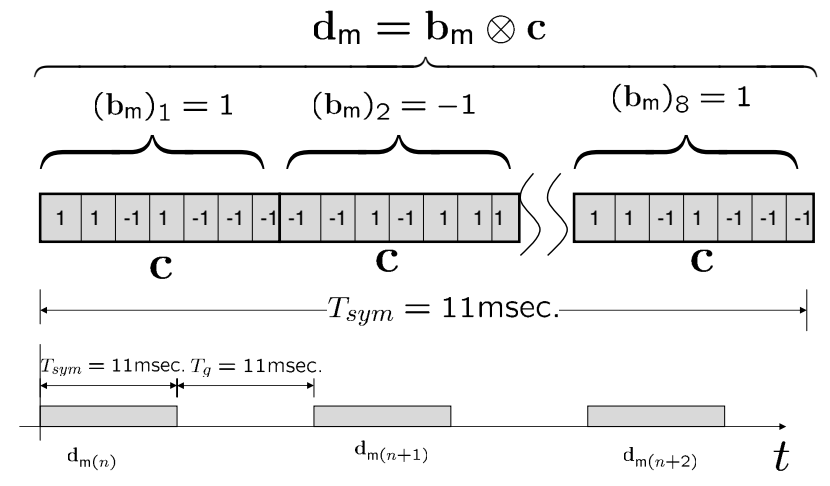

Fig. 1. Walsh/m-sequence signals.

on the DSP chip. In the transmit mode, the digital signal generated by the DSP is applied to a pair of DACs, one of which is dedicated to scaling the transmitted signal (for power control). The DAC output passes through a filter network identical to the receiver filter; this filter removes DAC harmonic artifacts. A Maxim MAX9703 class D power amplifier amplifies the signal to a maximum of 12 watts RMS, and a network consisting of a transformer and inductor cancel most of the transducer capacitance and approximately match the transducer impedance to the amplifier's design load. The preamp input remains coupled to the transducer during transmit but the power flow into the preamp is limited to prevent damage.

\section{CONCLUSIONS}

A design for an UWA modem was presented based on Walsh/m-sequence signaling. The proposed GMHTMP receiver significantly outperforms a RAKE detector at usable SNRs. The Matching Pursuits algorithm in particular is effective in estimating long, sparse underwater acoustic multipath channels, and is fairly robust to overparamterization.

Hardware implementation of the modem is underway in programmable DSP, and sea testing is anticipated by Fall 2005. Future research on the modem will include optimization of the Walsh signal dimension and spreading to increase data rates and obtain further robustness to frequency-selective multipath. Implementation in reconfigurable hardware will also be investigated as an alternative to DSP for lower power and accommodation of larger acoustic bandwidths and data rates. 


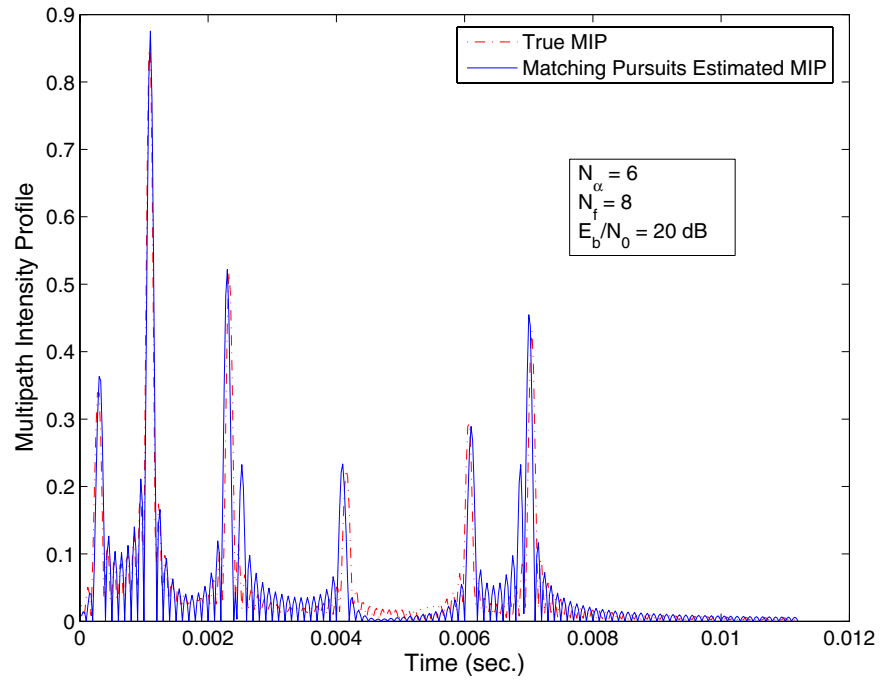

Fig. 2. Channel estimates at $E_{b} / N_{0}=20 \mathrm{~dB}, N_{\alpha}=6, N_{f}=8$

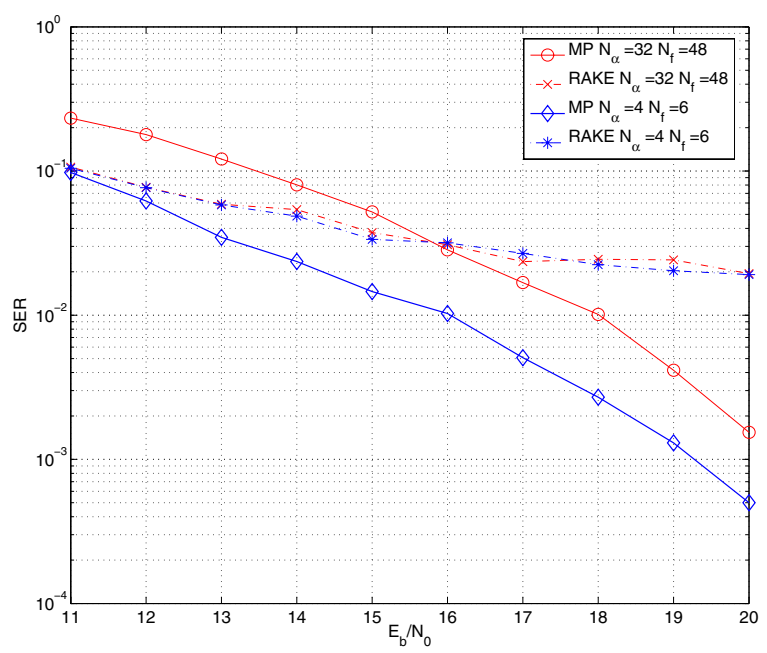

Fig. 3. SERs for $N_{\alpha}=6, N_{f}=8$ and $N_{\alpha}=32, N_{f}=48$.

\section{REFERENCES}

[1] D. Codiga, J. A. Rice, and P. Bogden, "Real-time wireless delivery of subsurface coastal circulation measurements from distributed intruments using networked acoustic modems," in Proceedings of MTS/IEEE Oceans 2000, vol. 1, pp. 575-582, 2000.

[2] J. G. Proakis, E. M. Sozer, J. A. Rice, and M. Stojanovic, "Shallow water acoustic networks," IEEE Communications Magazine, pp. 114-119, Nov. 2001.

[3] K. F. Scussel, J. A. Rice, and S. Merriam, "A new MFSK acoustic modem for operation in adverse underwater channels," in Proceedings of MTS/IEEE Oceans 97, vol. 2, (Halifax, N.S., Canada), pp. 247-253, 1997.

[4] A. Benson, J. Proakis, and M. Stojanovic, "Towards ro-

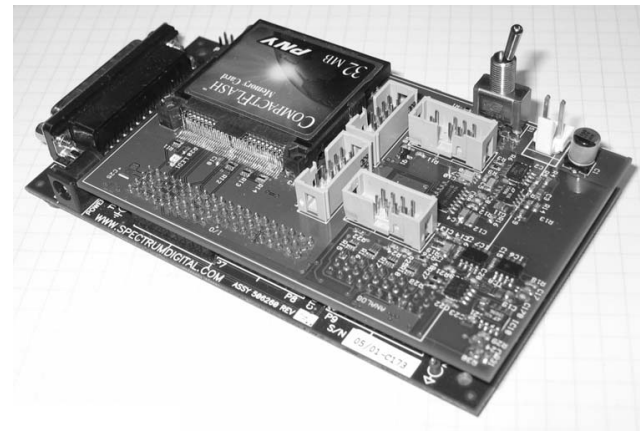

(a)

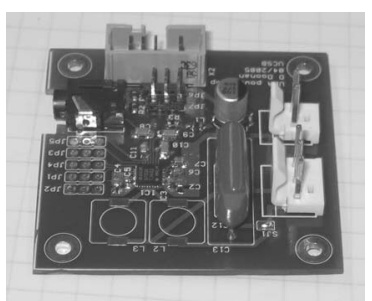

(b)

Fig. 4. (a) F2812 DSP with ADC/DAC. (b) Power amplifier

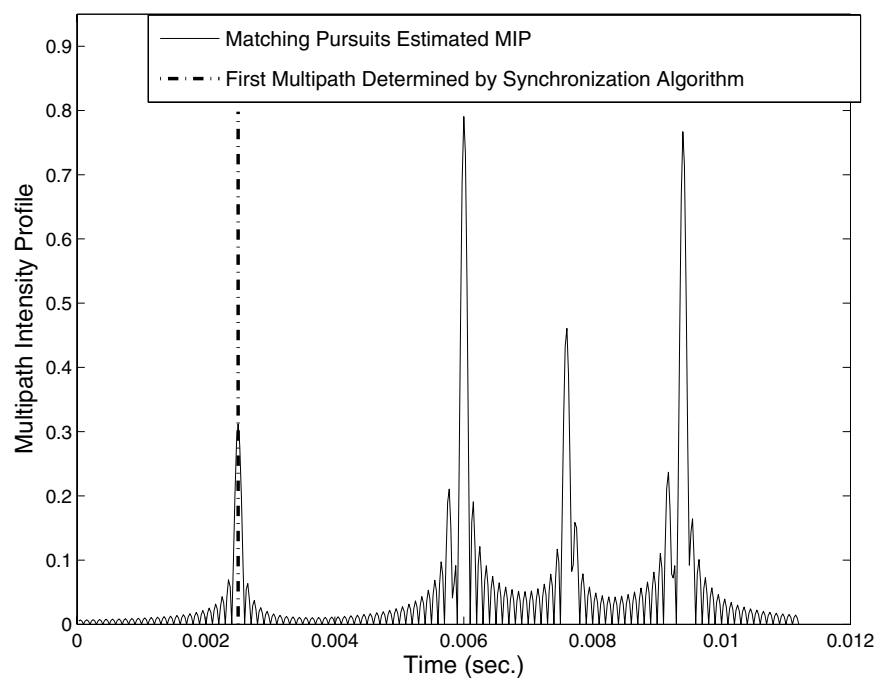

Fig. 5. Estimate of $l_{d}$ direct-path delay from synchronization algorithm 
bust adaptive acoustic communications," in Proceedings of MTS/IEEE Oceans 2000, pp. 1243-1284, 2000.

[5] M. Stojanovic, J. Catipovic, and J. Proakis, "Phase-coherent digital communications for underwater acoustic channels," IEEE Journal of Oceanic Engineering, vol. 19, pp. 100-111, Jan. 1994.

[6] Z. Zvonar, D. Brady, and J. Catipovic, "An adaptive decentralized multiuser receiver for deep-water acoustic telemetry," Journal of the Acoustical Society of America, vol. 101, pp. 23842387, April 1997.

[7] M. Stojanovic and L. Freitag, "Acquisition of direct-sequence spread-spectrum acoustic communication signals," in Proceedings of MTS/IEEE Oceans Conference 2003, pp. 279-286, 2003.

[8] L. Freitag, M. Stojavnovic, S. Singh, and M. Johnson, "Analysis of channel effects on direct-sequence and frequency-hopped spread-spectrum acoustic communication," IEEE Journal of Oceanic Engineering, vol. 26, pp. 586-593, Oct. 2001.

[9] D. B. Kilfoyle and A. Baggeroer, "The state of the art in underwater acoustic telemetry," IEEE Journal of Oceanic Engineering, vol. 25, pp. 4-27, January 2000.

[10] J. Fuchs, "Multipath time-delay detection and estimation," IEEE Transactions on Signal Processing, vol. 47, pp. 237-243, Jan. 1999.

[11] E. M. Sozer, J. G. Proakis, M. Stojanovic, J. A. Rice, A. Benson, and M. Hatch, "Direct sequence spread spectrum based modem for underwater acoustic communication and channel measurements," in Proceedings of MTS/IEEE Oceans Conference 1999, pp. 228-233, 1999.

[12] S. Kim and R. A. Iltis, "A Matching Pursuit/GSIC-based algorithm for DS-CDMA sparse channel estimation," IEEE Signal Processing Letters, vol. 11, pp. 12-15, Jan. 2004.

[13] S. F. Cotter and B. D. Rao, "Sparse channel estimation via matching pursuit with application to equalization," IEEE Transactions on Communications, vol. 50, pp. 374-377, March 2002. 